\title{
Intraligamentary Pregnancy with Recurrent Urine Retention: Case Report
}

\author{
Salma Abdi Mahmoud1,2*, Adam Munkaila², Ali M. Ussi'3, Chukwuma J. Okafor4, \\ Gumanga Solomon ${ }^{2}$, Mumuni Kareem ${ }^{4}$, Ali Said Yussuf ${ }^{1}$, Osman Saia ${ }^{5}$
}

\begin{abstract}
${ }^{1}$ Department of Obstetrics and Gynaecology, School of Health and Medical Sciences, The State University of Zanzibar, Zanzibar, Tanzania

${ }^{2}$ Department of Obstetrics and Gynaecology, University for Development Study, Tamale Teaching Hospital, Tamale, Ghana ${ }^{3}$ Department of Natural Sciences, The State University of Zanzibar, Zanzibar, Tanzania ${ }^{4}$ Department of Pathology and Biochemistry, School of Health and Medical Sciences, The State University of Zanzibar, Zanzibar, Tanzania

${ }^{4}$ Department of Obstetrics and Gynaecology, Korle Bu Teaching Hospital and University of Ghana Medical School, Accra, Ghana ${ }^{5}$ Department of Anaesthesia, Tamale Teaching Hospital, Tamale, Ghana
\end{abstract}

Email: *salma.mahmoud@suza.ac.tz

How to cite this paper: Mahmoud, S.A. Munkaila, A., Ussi, A.M., Okafor, C.J., Solomon, G., Kareem, M., Yussuf, A.S. and Saia, O. (2021) Intraligamentary Pregnancy with Recurrent Urine Retention: Case Report. Open Journal of Obstetrics and Gynecology, 11, 516-522.

https://doi.org/10.4236/ojog.2021.115048

Received: March 26, 2021

Accepted: May 11, 2021

Published: May 14, 2021

Copyright $\odot 2021$ by author(s) and Scientific Research Publishing Inc. This work is licensed under the Creative Commons Attribution International License (CC BY 4.0).

http://creativecommons.org/licenses/by/4.0/

\begin{abstract}
An intraligamentary pregnancy is an extremely rare form of ectopic pregnancy, with only a few cases reported. We report a 20-year-old woman who had an ongoing pregnancy at about 22 weeks gestation, consulted for recurrent urinary retention and severe lower abdominal pain of two days duration. Pelvic ultrasonography revealed an extrauterine gestation of 19 weeks and four days on the left adnexal region. Exploratory laparotomy was performed that found left intraligamentary pregnancy (unruptured) measured $20-25$ $\mathrm{cm}$ across, attached posteriorly to the bowel (sigmoid colon) and extended to the pouch of douglas impinging on the bladder base. Resection of ectopic pregnancy was successfully performed, urine retention resolved, and the patient was discharged with no postoperative complications. Early recognition of this rare form of ectopic gestation is of considerable importance because of the risk of a life-threatening haemorrhagic complication necessitating emergency surgical intervention.
\end{abstract}

\section{Keywords}

Intraligamentary Pregnancy, Urine Retention, Ultrasonography

\section{Introduction}

An intraligamentary pregnancy is a type of ectopic pregnancy developing within 
the broad ligament leaves [1] [2]. Intraligamentary gestation is boarded anteriorly and posteriorly by the leaves of the broad ligament, medially by the uterus and laterally by the pelvic side wall, inferiorly by the pelvic floor, and superiorly by the fallopian tubes. It is an abdominal pregnancy that develops retroperitoneally [3]. The prerequisites for the development of intraligamentary pregnancy are; the expanding gestational sac must split the oviduct precisely between the leaves of the broad ligament, the amnion at least must remain intact to permit the fetus to continue to grow in the extraperitoneal sac, and rupture must occur early enough so that the villi are capable of expanding their areas of nidation [4] [5]. The round ligament is usually found conspicuously on the anterior surface of the gestational sac. The placenta may erode beyond the tubal confines to invade the ovary, uterus, omentum, pelvic peritoneum, and adjacent viscera [6]. Intraligamentary pregnancy as a form of non tubal pregnancies, constitutes a small percentage $(<2 \%)$ of all ectopic pregnancies [7] [8]. Here we report a case of intraligamentary pregnancy with recurrent urine retention diagnosed and successful managed by exploratory laparotomy.

\section{Case Report}

We report the case of 20-year-old $\mathrm{G}_{4} \mathrm{P}_{1} \mathrm{~A}_{2}$, who presented with a history of recurrent urinary retention and severe lower abdominal pain of two days duration. She had an ongoing pregnancy at about 22 weeks gestation at the time of admission.

A week before her presentation at the district hospital, she had an onset of severe lower abdominal and waist pains managed with analgesics at a community clinic. Before her referral to the Tamale Teaching Hospital (TTH), she had recurrent urinary retention episodes relieved by urethral catheter passage anytime it occurred. She presented to the district hospital with another urinary retention episode three days after discharge on analgesics and antibiotics and was immediately referred to Tamale Teaching Hospital. She was admitted to having a urinary frequency, dysuria, straining on urination, and urgency before the urine retention. She also had episodes of scanty bleeding per vaginam.

Her past medical, surgical, and family histories were nil of significance. She was an unemployed single Junior high school graduate but in a stable relationship with 26 year old farmer. She did not take any habit-forming drugs. Her menarche was at 14 years, and she has a 28-day cycle with a duration of flow of 3 days. She had two-lifetime sexual partners and had not used any modern method of contraceptive before. Her first pregnancy was 4 years ago, carried to term, and had an uneventful spontaneous vaginal delivery. She also had two terminations of pregnancy due to social reasons (at 2 months each), a year before the current pregnancy. In the index pregnancy, she booked at 6 weeks at the community clinic.

Physical examination at the TTH showed a young lady who looked well, not pale, anicteric, not warm to touch. The pulse was 84 beats/minute; Blood pressure was $110 / 70 \mathrm{mmHg}$. The chest was clinically clear. Abdominal examination 
showed a 14-week size uterus with an enlarged soft and tender mass located in the left iliac fossa region, $5-10 \mathrm{~cm}$ in the widest diameter, that arose from the pelvis. She had bilateral renal angle tenderness. Pelvic examination revealed a healthy vulva and vagina. The cervix was $2 \mathrm{~cm}$ long, firm, anterior, and closed. Bimanual Uterine examination confirmed the 14-week size uterus with a mass of about $5 \times 8 \mathrm{~cm}$ at the left adnexal region separated from the uterus. Full Blood Count (FBC) was done, and it showed increased WBC with moderate leucocytosis. Urinalysis suggested urinary tract infection, but urine culture had no growth after 48 hours. The renal function test was normal. A radiologist specialist was consulted to perform Pelvic ultrasonography, and findings were that of an empty uterus, measuring $7.5 \times 5.68 \times 4.37 \mathrm{~cm}$ with thickened endometrium and no obvious intrauterine gestation, an extrauterine gestation of 19 weeks and 4 days with positive fetal heart tones, placenta, and adequate liquor were noted on the left adnexal region. A differential diagnosis of a viable extrauterine gestation was made.

The patient was thus counseled for exploratory laparotomy. The surgery findings were; Left intraligamentary pregnancy (unruptured), attached posteriorly to the bowel (sigmoid colon) and extended to the pouch of douglas impinging on the bladder base. It was also attached to the omentum with huge tortuous vessels on the left broad ligament's anterior wall. The gestational sac measures about $20-25 \mathrm{~cm}$ across. The right tube and both ovaries appeared normal. The left tube appeared enlarged with distortions at the inferior part. The uterus was bulky and empty, as shown in Figure 1. The broad ligament's anterior layer was incised and baby delivered by breech extraction, the cord was cut short, and placenta was left within. The fetus was alive (male fetus that died soon after delivery as it was being sent to Neonate Intensive Care Unit), it weighed $550 \mathrm{~g}$, no gross anomalies as shown in Figure 2.

Post-operative period was uneventful. The urethral catheter was removed after 24 hours of surgery. She did not develop urine retention again. She continued the antibiotics for one weeks after surgery. She was followed up postoperatively with serial serum $\beta$-HCG which are shown in Table 1 and Figure 3. Patient's

Table 1 . Shows weekly regression of data $\beta$-HCG post laparotomy.

\begin{tabular}{cc}
\hline \multirow{2}{*}{ Weeks } & Weekly Regression of Serum $\beta$-HCG \\
\cline { 2 - 2 } & Serum $\beta$-HCG mlU/ml \\
\hline 2 & 41323 \\
4 & 13235 \\
10 & 3820 \\
20 & 1860 \\
24 & 650 \\
28 & 80 \\
32 & 0.5 \\
\end{tabular}




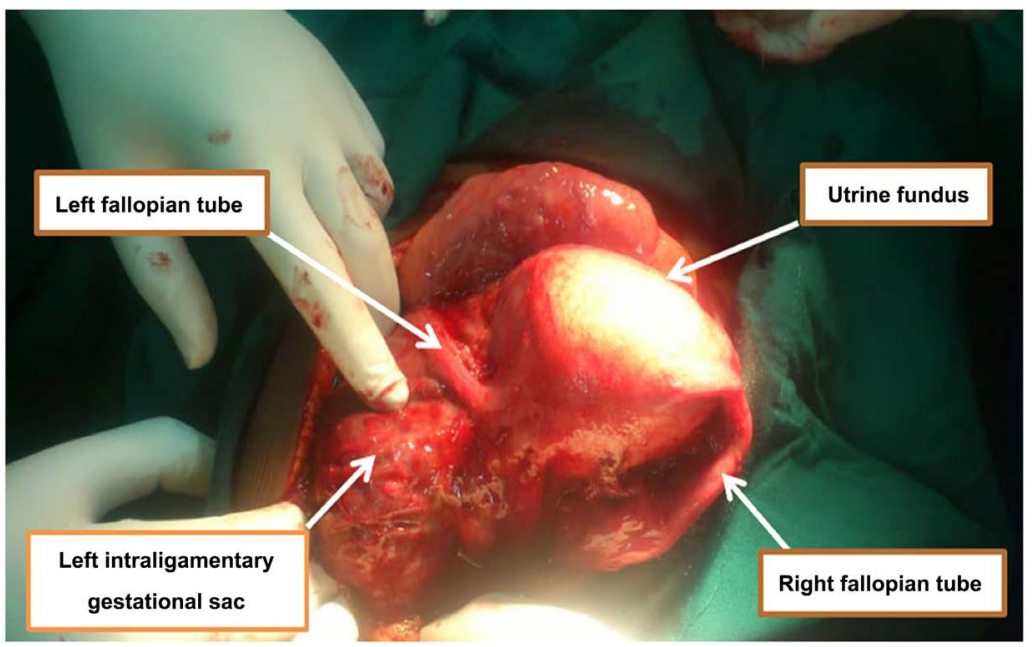

Figure 1. Shows left intraligamentary pregnancy.

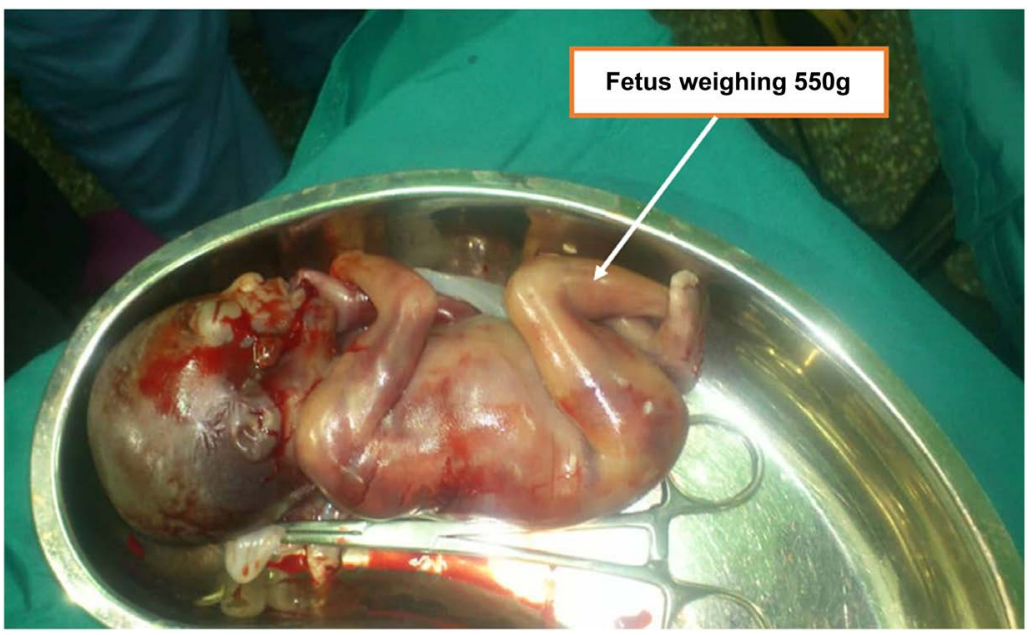

Figure 2. Shows fetus weigh $550 \mathrm{~g}$.

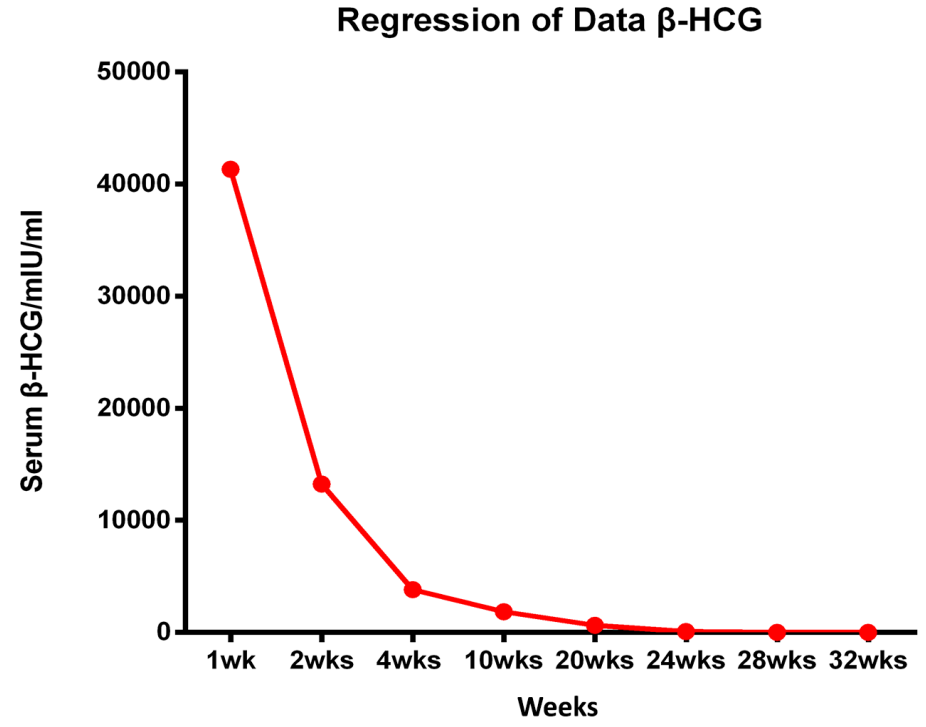

Figure 3. Shows weekly regression of data $\beta$-HCG post laparotomy. 
general condition was satisfactory, and all the systemic and pelvic examinations carried out were normal. Repeated abdomen and pelvic scan after five weeks post-delivery did not reveal any abnormalities.

\section{Discussion}

An intraligamentary pregnancy, also known as broad ligamentary ectopic pregnancy, is an extremely rare condition and is said to be an abdominal pregnancy implanted between the two layers of the broad ligament [9] [10]. It is believed to result from rupture of tubal pregnancy on the mesosalpingeal border [9]. The findings on our case that the inferior portion of the left tube appeared distorted and enlarged concur with the existing literature.

Clinical diagnosis of intraligamentary pregnancy can be very difficult and needs a high index of suspicion. Not only does it pose a diagnostic problem to the clinician, but it also possesses a profound therapeutic difficulty giving the peculiar nature of its localization. It may never even be diagnosed until at caesarean section [11]. Intraligamentary pregnancy has been reported to occur in one out of 245 ectopic pregnancies [2], in one out of 613 ectopic gestations, whereas others reported the frequency of once in every 183,900 pregnancies [5] [12].

A study by SA. Obed et al. 2006 at Korle-bu Teaching Hospital in Ghana on ectopic pregnancies, found the incidence of tubal ectopic pregnancy to be 32.9 per 1000 deliveries and 5.43\% of this formed unruptured cases [13]. Likewise, Petter Baffoe and colleagues at Boligatanga Regional hospital in Ghana in 2011 reported on term abdominal pregnancy with healthy new born weighing 2.3 killograms [14]. Our case was the first reported case of intraligamentary pregnancy in Tamale Teaching Hospital (TTH), which occurred out of 59 ectopic pregnancies diagnosed between 2014 and 2018.

This particular case was peculiar in that she presented with recurrent urine retention and constipation. These pressure symptoms could be due to the impaction of the gestational on the pouch of Douglas compressing the bladder and the large bowel that was found intraoperatively. An ultrasound could be helpful in the diagnosis of intraligamentary pregnancy. However, ultrasound has been shown to miss $50 \%$ of the diagnosis of cases of intra-abdominal pregnancy [15]. In our case, only one out of the four ultrasound scans revealed an extrauterine gestation. This was done by an experienced specialist radiologist. Magnetic Resonance Imaging and Computed Tomography are the other better radiological means of diagnosis [12].

Therefore, the diagnosis would pose a problem to the health facilities in which human resource is lacking in imaging and diagnostics, especially in the deprived northern sector of Ghana, where there are very few Radiologists. Thus, it is imperative to use our clinical suspicion very well to diagnose the condition and to intervene timeously to avert life-threatening complications.

In our estimation, the serious complications in this condition are severe haemorrhage; from intra-abdominal rupture of the gestational sac, intraoperative 
and immediate post-operative bleeding from the placental site. Generally, it is recommended that the cord is cut short and the placenta is left in situ, and postoperatively, the patient's $\beta$-HCG is monitored [5] [16], as it was done in our case.

\section{Conclusion}

An intraligamentary pregnancy is a rare but life-threatening obstetric condition with high maternal morbidity-mortality. Diagnosis is extremely difficult, and a high index of suspicion is needed. Besides, an experienced radiologist may be helpful, especially at peripheral health facilities. An appropriate preoperative evaluation and surgical technique are imperative for a favorable outcome.

\section{Statement}

The patient has given her consent for the case report to be published.

\section{Conflicts of Interest}

The authors report no conflict of interest.

\section{References}

[1] Carusi, D. (2019) Pregnancy of Unknown Location: Evaluation and Management. Seminars in Perinatology, 43, 95-100. https://doi.org/10.1053/j.semperi.2018.12.006

[2] Cordero, D.R., Adra, A., Yasin, S. and O’Sullivan, M.J. (1994) Intraligamentary Pregnancy. Obstetrical \& Gynecological Survey, 49, 206-209. https://doi.org/10.1097/00006254-199403000-00026

[3] Ameer, M.A., Fagan, S.E., Sosa-Stanley, J.N. and Peterson, D.C. (2021) Anatomy, Abdomen and Pelvis, Uterus. http://www.ncbi.nlm.nih.gov/pubmed/29262069

[4] Ade-Ojo, I.P., Akintayo, A.A., Afolayan, J.M., Aduloju, O.P. and Olagbuji, B.N. (2016) Intraligamentary Extrauterine Pregnancy Delivered at Term: A Case Report and Review of Literature. African Journal of Reproductive Health, 20, 104-108. https://doi.org/10.29063/ajrh2016/v20i1.11

[5] Gao, J., Liu, L., Fang, J. and Wang, Y. (2021) Broad Ligament Pregnancy: A Case Series and Literature Review. Journal of Obstetrics and Gynaecology Research, 47, 442-445. https://doi.org/10.1111/jog.14564

[6] Suzuki, S., Takahashi, T., Ota, K., Nishimura, K., Fukase, M., Watanabe, N., et al. (2019) Successful Laparoscopic Treatment of an Abdominal Pregnancy Implanted in the Utero-Ovarian Ligament: A Case Report of a Rare Form of Ectopic Pregnancy. Journal of Obstetrics and Gynaecology, 39, 579-580. https://doi.org/10.1080/01443615.2018.1534816

[7] Rokhgireh, S., Gorginzadeh, M., Mehdizadehkashi, A., Tahermanesh, K. and Alizadeh, S. (2021) Broad Ligament Pregnancy in the Presence of an Intrauterine Contraceptive Device: A Case Report. International Journal of Surgery Case Reports, 79, 421-423. https://doi.org/10.1016/j.ijscr.2021.01.080

[8] Poole, A., Haas, D. and Magann, E.F. (2012) Early Abdominal Ectopic Pregnancies: A Systematic Review of the Literature. Gynecologic and Obstetric Investigation, 74, 249-260. https://doi.org/10.1159/000342997

[9] Vierhout, M.E. and Wallenburg, H.C. (1985) Intraligamentary Pregnancy Resulting 
in a Live Infant. American Journal of Obstetrics \& Gynecology, 152, 878-879. https://doi.org/10.1016/S0002-9378(85)80081-8

[10] Chouinard, M., Mayrand, M.-H., Ayoub, A., Healy-Profitós, J. and Auger, N. (2019) Ectopic Pregnancy and Outcomes of Future Intrauterine Pregnancy. Fertility and Sterility, 112, 112-119. https://doi.org/10.1016/j.fertnstert.2019.03.019

[11] Audifred Salomón, J.R., Herrera Ortiz, A., González Medrano, M.G. and Estrada Rivera, S.F. (2013) Ectopic Intraligamentary Pregnancy. Revista de Ginecología y Obstetricia de México, 81, 211-214.

[12] Azhar, E., Green, L., Mohammadi, S. and Waheed, A. (2020) Ruptured Right Broad Ligament Ectopic Pregnancy in a Patient with Prior Right Salpingo-Oophorectomy: A Case Report. Cureus, 12, Article No. e8276. https://doi.org/10.7759/cureus.8276

[13] Asah-Opoku, K., Oppong, S.A., Ameme, D.K., Nuamah, M.A., Mumuni, K., Yeboah, A.O., et al. (2019) Risk factors for Ectopic Pregnancy among Pregnant Women Attending a Tertiary Healthcare Facility in Accra, Ghana. International Journal of Gynecology \& Obstetrics, 147, 120-125. https://doi.org/10.1002/ijgo.12928

[14] Rudra, S., Gupta, S., Taneja, B.K. and Garg, M. (2013) Full-Term Broad Ligament Pregnancy. Case Reports, 2013, Article ID: bcr2013010329. https://doi.org/10.1136/bcr-2013-010329

[15] Kirk, E. (2012) Ultrasound in the Diagnosis of Ectopic Pregnancy. Clinical Obstetrics and Gynecology, 55, 395-401. https://doi.org/10.1097/GRF.0b013e31824e35fe

[16] Sassi, A., Dimassi, K., Ben Slama, S., Triki, A. and Lahmar, A. (2018) A Broad Ligament Pregnancy Successfully Managed by Laparoscopy. Journal of Obstetrics and Gynaecology, 38, 423-424. https://doi.org/10.1080/01443615.2017.1342231 